\title{
Mechanical properties of nanotube sheets: Alterations in joint morphology and achievable moduli in manufacturable materials
}

L. Berhan and Y. B. Yi

Department of Mechanical Engineering, University of Michigan, Ann Arbor, Michigan 48109

\author{
A. M. Sastrya) \\ Department of Mechanical Engineering and Department of Biomedical Engineering, \\ University of Michigan, Ann Arbor, Michigan 48109
}

\author{
E. Munoz, M. Selvidge, and R. Baughman \\ UTD NanoTech Institute, University of Texas at Dallas, Dallas, Texas 75083
}

(Received 4 August 2003; accepted 27 January 2004)

\begin{abstract}
Nanotube sheets, or "bucky papers," have been proposed for use in actuating, structural and electrochemical systems, based in part on their potential mechanical properties. Here, we present results of detailed simulations of networks of nanotubes/ropes, with special emphasis on the effect of joint morphology. We perform detailed simulations of three-dimensional joint deformation, and use the results to inform simulations of two-dimensional (2D) networks with intertube connections represented by torsion springs. Upper bounds are established on moduli of nanotube sheets, using the 2D Euler beam-network simulations. Comparisons of experimental and simulated response for HiPco-nanotube and laser-ablated nanotube sheets, indicate that $\sim 2-30$-fold increases in moduli may be achievable in these materials. Increasing the numbers of interrope connections appears to be the best target for improving nanotube sheet stiffnesses in materials containing straight segments. (C) 2004 American Institute of Physics. [DOI: 10.1063/1.1687995]
\end{abstract}

\section{INTRODUCTION}

The potential mechanical properties of nanotube sheets or "bucky papers" have motivated proposal of their use in many applications, including actuators, ${ }^{2,3}$ capacitors, electrodes, and field emission devices. The moduli of these porous fibrous materials are necessarily less than those of individual nanotubes or ropes, but without realistic bounds on the properties of the sheets themselves it is difficult to define an "optimized" sheet. Measured Young's moduli in conventionally produced nanotube sheets have hovered around 2 $\mathrm{GPa},{ }^{4}$ or approximately $0.2 \%$ of the modulus of singlewalled nanotubes. ${ }^{5}$

Newer manufacturing approaches have produced moderate improvements in modulus and strength of these porous assemblies (Table I). Shreekumar et al. ${ }^{6}$ obtained a Young's modulus of $8 \mathrm{GPa}$ for nanotube films manufactured from a single-walled carbon nanotube solution in oleum. Coleman et al. ${ }^{4}$ reported moduli of approximately $7 \mathrm{GPa}$ for polymerintercalated nanotube sheets. Magnetic alignment ${ }^{7-9}$ has also produced improvements. Li et al. ${ }^{9}$ reported Young's moduli of 24 and $60 \mathrm{GPa}$, respectively, for two types of these ribbons. However, even these improved moduli are less than $10 \%$ of those of the constituent nanotubes.

Nanotube sheets (e.g., the material shown in Fig. 1) are manufactured using the same basic approach, ${ }^{10}$ namely, via filtration of a suspension of single-walled nanotubes (SWNTs). The resulting sheets have a laminar structure [Fig. 1(a)]; orientations of nanotube ropes are random within the

\footnotetext{
a) Author to whom correspondence should be addressed; electronic mail: amsastry@umich.edu
}

plane of the sheet [Fig. 1(b)]. Nanotube sheets thus comprise fibrous networks, wherein the connective elements are ropes of 100-500 single-walled carbon nanotubes. ${ }^{5}$

In this work we use two-dimensional (2D) beam network simulations to develop realistic upper bounds on the Young's modulus of nanotube sheets. Our objectives were threefold:

(1) to develop reasonable models for load transfer within nanotube sheets, incorporating recent work on the importance of joint morphology;

(2) to use the models to develop bounds on likely properties of nanotube sheets, and

(3) to compare experimental moduli with the models, to determine the likely improvements possible in nanotube sheet properties.

\section{A. Construction of 2D fiber network models}

The mechanics of paper and other fibrous materials has been studied by many researchers, including Cox,${ }^{11}$ who developed an upper bound on moduli of similar assemblies. However, the fibers in this model were assumed to have negligible flexural rigidity, since they were assumed to be fully supported. Thus, the micromechanical assumptions are not readily transferrable to unsupported nanotube ropes. Moreover, the model did not address variable joint properties, a key interest of the present work.

Other workers (e.g., Lu and Carlsson) ${ }^{12}$ have specifically modeled load transfer within porous systems, such as papers, following early work by Kallmes and Corte. ${ }^{13}$ The latter authors studied the statistical geometry of idealized twodimensional fiber networks comprised of randomly distributed fibers, as defined by their centerpoints and orientations. 
TABLE I. Mechanical properties of carbon nanotube sheets and ribbons, from selected references.

\begin{tabular}{|c|c|c|c|c|c|c|c|}
\hline \multicolumn{2}{|c|}{ Mechanical property } & \multicolumn{3}{|c|}{ Physical properties } & \multicolumn{2}{|c|}{ Experimental details } & \multirow[b]{2}{*}{ Reference } \\
\hline $\begin{array}{l}\text { Young's } \\
\text { modulus } \\
(\mathrm{GPa})\end{array}$ & $\begin{array}{c}\text { Tensile strength } \\
(\mathrm{MPa})\end{array}$ & $\begin{array}{c}\text { Nanotube synthesis } \\
\text { method }\end{array}$ & $\begin{array}{l}\text { Average } \\
\text { tube } \\
\text { diameter } \\
(\mathrm{nm})\end{array}$ & $\begin{array}{l}\text { Average } \\
\text { rope } \\
\text { diameter } \\
(\mathrm{nm})\end{array}$ & Test method & $\begin{array}{l}\text { Sample } \\
\text { size }\end{array}$ & \\
\hline $\begin{array}{l}24 \\
\text { (as grown) } \\
60 \\
\text { (graphitized) }\end{array}$ & $\cdots$ & $\begin{array}{l}\text { Ribbons of magnetically } \\
\text { aligned nanotubes } \\
\text { synthesized by } \\
\text { decomposition of } \\
\text { hydrocarbons using } \\
\text { chemical vapor } \\
\text { deposition method }\end{array}$ & $\cdots$ & $\cdots$ & $\begin{array}{l}\text { Tensile test } \\
\text { using specially } \\
\text { designed } \\
\text { stress-strain } \\
\text { puller }\end{array}$ & $\begin{array}{l}50-140 \mathrm{~mm} \\
\times 4-40 \mathrm{~mm} \\
\times 100 \mathrm{~mm}\end{array}$ & 9 \\
\hline 8 & 30 & $\begin{array}{l}\text { Dispersion of HiPco } \\
\text { SWNTs in oleum used to } \\
\text { form film }\end{array}$ & 0.8 & $10-50$ & $\begin{array}{l}\text { Tensile tests } \\
\text { using } \\
\text { Rheometrics } \\
\text { RSA III at } \\
0.72 \% / m i n \\
\text { strain rate }\end{array}$ & $\begin{array}{l}2 \mathrm{~mm} \times \\
25 \mathrm{~mm} \times \\
0.015 \mathrm{~mm}\end{array}$ & 6 \\
\hline 6.9 & 57 & $\begin{array}{l}\text { HiPco sheets } \\
\text { intercalated with } \\
\text { polymer }\end{array}$ & 0.8 & $10-50$ & $\begin{array}{l}\text { Tensile tests } \\
\text { using a Perkin } \\
\text { Elmer DMA7e }\end{array}$ & $\begin{array}{l}2 \mathrm{~mm} \times \\
20 \mathrm{~mm} \times \\
0.040 \mathrm{~mm}\end{array}$ & 4 \\
\hline 1.2 & $\cdots$ & $\begin{array}{l}\text { Laser vaporization } \\
\text { method }\end{array}$ & $\cdots$ & $\cdots$ & $\cdots$ & $\cdots$ & 2 \\
\hline 2.3 & 6.29 & HiPco process & 0.8 & $10-50$ & $\begin{array}{l}\text { Tensile tests } \\
\text { using a Perkin } \\
\text { Elmer DMA7e }\end{array}$ & $\begin{array}{l}2 \mathrm{~mm} \times \\
20 \mathrm{~mm} \times \\
0.040 \mathrm{~mm}\end{array}$ & 4 \\
\hline 1.1 & 17.7 & $\begin{array}{l}\text { HiPco process } \\
\text { (annealed sheet) }\end{array}$ & 0.8 & $\sim 10$ & $\begin{array}{l}\text { Tensile tests } \\
\text { using a Perkin }\end{array}$ & $\begin{array}{l}\text { 1mmwide } \\
\text { strips }\end{array}$ & (Present article) \\
\hline 4 & 32.3 & $\begin{array}{l}\text { HiPco process } \\
\text { (unannealed sheet) }\end{array}$ & 0.8 & $\sim 10$ & Elmer DMA7e & & \\
\hline $\begin{array}{l}1.5 \\
2\end{array}$ & 13.525 .4 & $\begin{array}{l}\text { Laser ablation method } \\
\text { (annealed sheets) }\end{array}$ & 1.36 & $\sim 10$ & & & \\
\hline 2.7 & 33.2 & $\begin{array}{l}\text { Laser ablation method } \\
\text { (unannealed sheet) }\end{array}$ & 1.36 & $\sim 10$ & & & \\
\hline
\end{tabular}

Kallmes and Corte ${ }^{13}$ modeled elastic behavior of fibrous networks using three statistical quantities: the total number of fiber crossings or intersections in the sheet, the mean number of crossings per fiber, and the mean free fiber length, defined as the distance between successive intersections along a fiber. We note that a fiber "crossing" is a statistical quantity, which may or may not result in a fiber-fiber "bond" in practice, depending on manufacturing process. The importance of this distinction is described in greater detail in the discussion section. Nonetheless, each of the three statistical parameters must be determined experimentally, or specified during manufacturing. In papers, for example, the "staple length," or initial cellulose fiber length, is a controllable parameter.

Unlike paper, however, the exact staple lengths of individual ropes within nanotube sheets are presently unknown, though they are clearly longer than the frame boundaries of scanning electron microscopy (SEM) images typically used to obtain other geometrical parameters. Models of fibrous arrays as 2D networks of infinite staple fibers have been previously developed, and provide some readily adaptable results for defining the geometry of such systems. For example, Goudsmit ${ }^{14}$ first studied the problem of infinite lines randomly distributed in a plane. Miles, ${ }^{15,16}$ also motivated by the study of the structure of paper, later examined the statis- tical properties of the polygons formed by a system of infinite lines randomly distributed in a plane.

\section{B. Bond behavior in fibrous, porous materials}

As discussed by Sastry et al., ${ }^{17}$ the mechanical response of a large class of porous, fibrous materials is largely determined by the morphology and composition of the joints among network elements. Stiffnesses of such materials have been satisfactorily predicted using analyses of $2 \mathrm{D}$ networks of both Euler and Timoshenko beam elements, ${ }^{18,19}$ and semiempirical models of joint response. For example, in fibrous substrates used in nickel/metal hydride batteries, simple 2D torsion springs were found to satisfactorily model joint compliance. Also, equivalence between a "compliant zone" around connected beams, and a torsion spring-jointed assembly has been demonstrated. ${ }^{20}$ Thus, the properties of a torsion spring-joined assembly can be specified by observed geometric features, and/or properties of the joint material.

Separate examination of joint material properties and geometric properties requires modeling the full, threedimensional (3D) joint morphology. ${ }^{21}$ For example, the present authors recently used a shear factor ${ }^{21}$ to scale local joint stresses in a 2D simulation, per calculated stress con- 


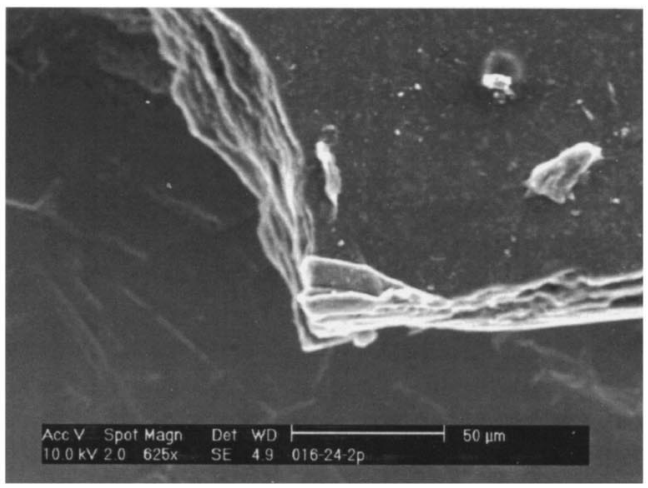

(a)

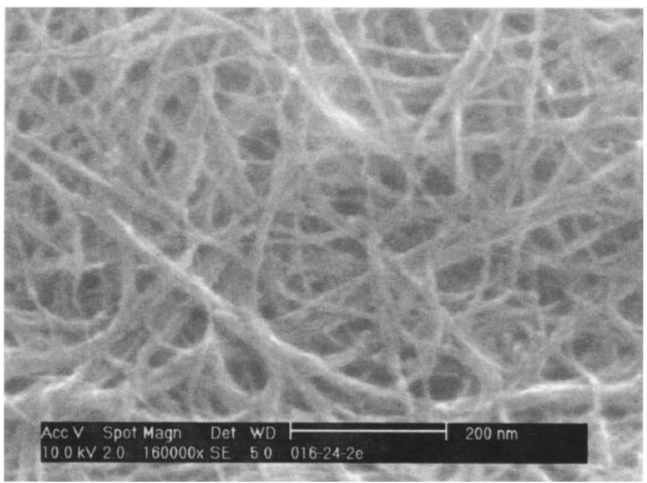

(b)

FIG. 1. SEM images of nanotube sheets, at magnifications (a) $625 \times$ and (b) $160000 \times$. Nanotubes were synthesized using the HiPco process.

centrations at a 3D fiber connection, and found that joint morphology is important in strength, as well as stiffness.

\section{MODEL DEVELOPMENT}

Our approach in modeling nanotube sheets builds upon our prior finding that joint morphology and response are critical to network response. Our general approach to the present problem is shown schematically in Fig. 2. The nanotube sheet [Fig. 2(a)] is first idealized as a network of random, infinite lines of constant, nonzero thickness [Fig. 2(b)]. This network of thick lines can be represented by an equivalent network of one-dimensional (1D) beam elements of "infinite" length having the same governing geometric property of mean segment aspect ratio [Fig. 2(c)]. This model is further reduced to a network of finite length beam elements randomly distributed in a finite simulation window [Fig. 2(d)], wherein periodic boundary conditions are imposed and non load-bearing ends are removed [Fig. 2(e)]. Torsion springs are used to model the joints in the network. We use the results of $3 \mathrm{D}$ joint models, that are believed to be morphologically similar to the actual connections [Fig. 2(f)], to select torsion spring constants for 2D simulations.

In our simulations, we necessarily use beams of finite length, randomly distributed in a finite simulation window. Bond density (number of intersections/unit area) and segment lengths (obtained from image analysis of nanotube

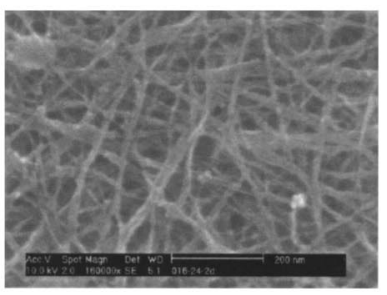

(a)

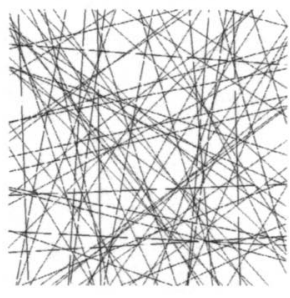

(c)

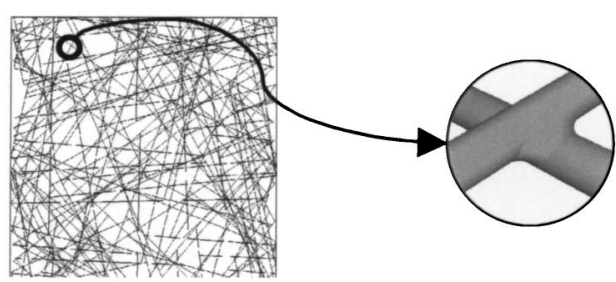

(e) (f)

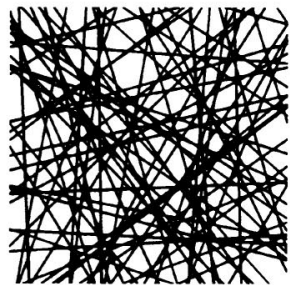

(b)

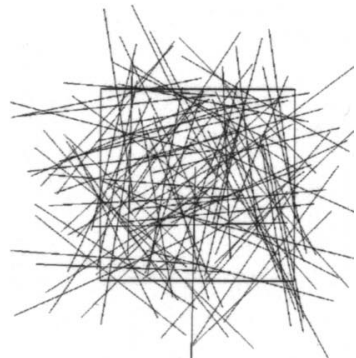

(d)
FIG. 2. Schematic of modeling approach to determine the modulus of a typical nanotube sheet. Initially, the sheet is imaged via SEM to obtain the mean nanorope diameter and the area fraction of nanotubes (a), whereupon the sheet is then idealized as a network of thick lines of 'infinite' length (b). Using Eq. (11) the mean segment aspect ratio can then be calculated. This parameter is then matched, using Eq. (14) to a network of infinitesimally thin lines used to simulate network behavior (c). For a sufficiently large number of infinitesimally thin lines, a network of finite lines of infinitesimal width randomly distributed in a finite simulation window can be used to simulate the array (d). Finally, finite element analysis is performed on the reduced network (e), informed by 3D finite element joint models (f).

sheets) are used to generate these finite networks. Several parameters are determined or estimated from image analysis, as described in the following sections.

\section{A. Network geometry}

The critical parameters of mean number of crossings per fiber and the mean segment length can be calculated given the total number of fiber crossings, $N_{c}$. Kallmes and Corte ${ }^{13}$ expressed the total number of fiber crossings in a random $2 \mathrm{D}$ network of area $A$, consisting of straight lines of negligible width, as

$$
N_{c}=\frac{\left(N_{f} L_{f}\right)^{2}}{A \pi},
$$

where $N_{f}$ and $L_{f}$ are the number of fibers and the average fiber length, respectively. Miles ${ }^{15}$ defined $\tau$, the average length of 1D lines per unit area, as

$$
\tau=\frac{N_{f} L_{f}}{A}
$$

and expressed the total number of fiber crossings 


$$
N_{c}=\frac{A \tau^{2}}{\pi} .
$$

Substitution of Eq. (2) in Eq. (3) confirms that the expressions of Kallmes and Corte ${ }^{13}$ and Miles ${ }^{15}$ are indeed equivalent for 1D lines.

Considering a random network of thick lines of width $w$, Miles ${ }^{15}$ developed an expression for the fraction of the plane not covered by thick lines, from which we can derive an expression for the mean fraction of the sheet covered by thick lines. This value, the coverage or area fraction $H$, is given by

$$
H=1-e^{-\tau w},
$$

where $\tau$ is defined in Eq. (2). It follows that the number of fiber crossings is given by

$$
N_{c}=\frac{A \log ^{2}(1-H)}{\pi w^{2}} .
$$

The details of the derivation of this expression are reserved for discussion in a companion paper focusing on statistics of random networks. ${ }^{22}$

Corte $^{23}$ also presented an expression for $N_{c}$, for a network of area $A$, comprised of randomly distributed and oriented thick fibers based on the mean coverage of the sheet. The coverage was defined as ratio of the total area of fibers to the sheet area. Corte's ${ }^{23}$ expression for coverage differs from that of Miles, ${ }^{15}$ since Miles ${ }^{15}$ explicitly accounted for the overlap area of crossing fibers into account. For $H$ $<10 \%$, the expressions differ by less than $5 \%$, but for large area fractions the difference is more significant, with a difference at $H=25 \%$, for example, of approximately $12 \%$. Here, we adopt the approach of Miles, ${ }^{15}$ since it fully accounts for overlap area.

Derivation of other geometrical parameters follows. For a random fiber network, the mean number of fiber crossings per fiber is

$$
\bar{c}=\frac{2 N_{c}}{N_{f}},
$$

and the mean segment length, $\bar{L}_{s}$ is given by

$$
\bar{L}_{s}=\frac{L_{f}}{\bar{c}} .
$$

By substituting Eq. (5) into Eq. (6), Eq. (7) can be rewritten as

$$
\bar{L}_{s}=-\frac{\pi w}{2 \log (1-H)} .
$$

The mean segment length for 1D lines is obtained by substituting Eq. (1) in Eq. (6) and rewriting Eq. (7) as

$$
\bar{L}_{s}=\frac{\pi A}{2 N_{f} L_{f}} .
$$

Average rope diameter can be found from image analysis, though it is difficult to obtain values of $N_{f}$ and $L_{f}$ in this way. We are able, however, to estimate the coverage $H$ from image analysis using a thresholding function to calculate the proportion of a single layer of the sheet covered by nanotubes. Using SEM images for this purpose introduces a fieldof-depth error, but by adjusting the thresholding level, it is possible to use SEM images to reasonably distinguish ropes lying in the top layer from those in other layers.

For uncorrelated fiber centers, we can assume that formation of a random network of a large number of fibers is a 2D Poisson process. This allows derivation of distribution functions for all other geometric characteristics of the network. For example, the segment length distribution is given by

$$
f\left(L_{s}\right)=\frac{1}{\bar{L}_{s}} \exp \left(-\frac{1}{\bar{L}_{s}} L_{s}\right),
$$

where $\bar{L}_{s}$ is both the mean segment length and the standard deviation.

If the total length of lines per unit area of a random network is constant, the averages of the geometric features of the resultant interior polygons (e.g., area, number of sides, etc.) are independent of the linewidth. ${ }^{15}$ Thus the mean segment length for a network of infinite thick lines is the same as that of a network of 1D lines of finite length, provided that the total length of beams is the same for both networks.

\section{Determination of the minimum number of fibers required in simulations}

To determine the minimum number of fibers per unit area required in our simulations, random networks were generated as shown in Fig. 3, and as described in earlier work. ${ }^{18}$ Briefly, beam elements with random orientations and centerpoints were distributed within the simulation window. Periodic boundary conditions were imposed and the nonloadbearing beam segments were removed. A square simulation window was used for each network, with side lengths $L_{u}$ $=L_{f}=1$.

Twenty network "realizations," of 10-120 fibers each, were generated for each value of $N_{f}$ examined. Resulting mean segment lengths versus $N_{f}$ are shown in Fig. 4, with error bars representing $\pm 1 \sigma$ (assuming normally distributed data), along with the analytical result for 1D lines [Eq. (9)], with $L_{f}=1$ and $A=1$. For $N_{f} \geqslant 60$, simulated mean segment lengths were within 5\% of the analytical predictions. Based on these findings, and practical limits of computing time, our simulations were run with $N_{f}=200$.

\section{Determination of beam diameter for finite element analysis}

For the experimental materials, the average rope diameter, $d$ and area fraction, $H$ were determined via image analysis, from which segment lengths were calculated. Mean segment aspect ratio was calculated by considering fibers of circular cross section having diameter $d$; replacing $w$ with $d$ in Eq. (8), we have

$$
\frac{\bar{L}_{s}}{d}=-\frac{\pi}{2 \log (1-H)} \text {. }
$$

A comparison of this result with that of Corte $^{23}$ is given in Fig. 5. 


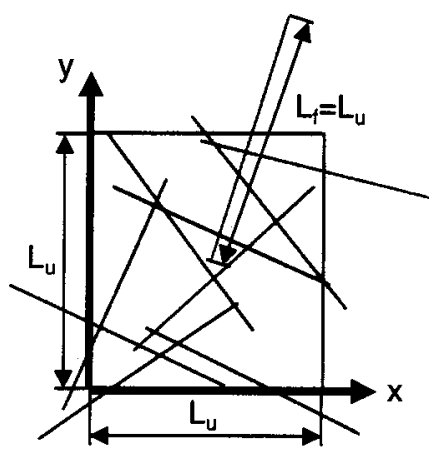

(a)

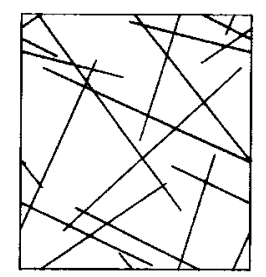

(b)

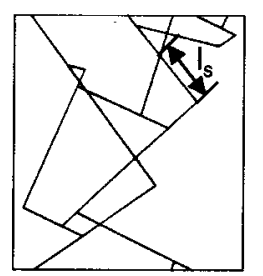

(c)

FIG. 3. Methodology used for generating random beam networks used in finite element analysis, including (a) random distribution of beams, using centerpoints and orientations, (b) imposition of periodic boundary conditions on the arrays, and (c) removal of non load-bearing beam segments.

Equation (9) gives the mean segment length for a network of lines of negligible thickness. For our finite element analyses, these segments were modeled as beam elements of diameter $d$ and aspect ratio

$$
\frac{\bar{L}_{s}}{d}=\frac{\pi A}{2 N_{f} L_{f} d} .
$$

Setting Eq. (11) $\left(\bar{L}_{s} / d\right.$ for an actual material) equal to Eq. (12) $\left(\bar{L}_{s} / d\right.$ for a simulation of beam elements) yields

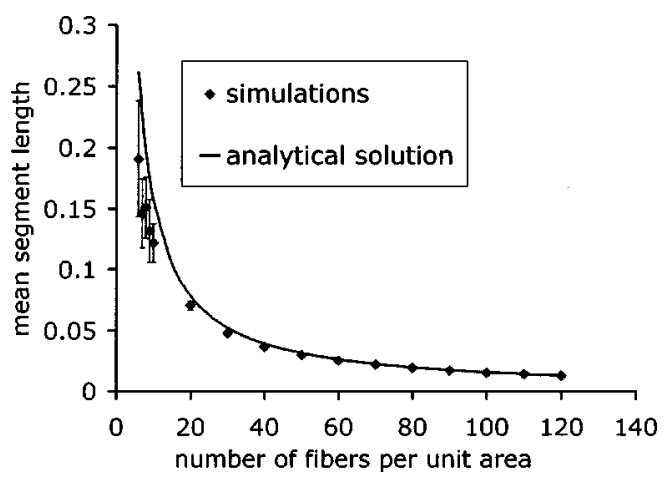

FIG. 4. Simulated and analytical results [Eq. (9)] for mean segment length vs number of fibers per unit area for fibers of negligible width and unit length distributed randomly in a unit cell.

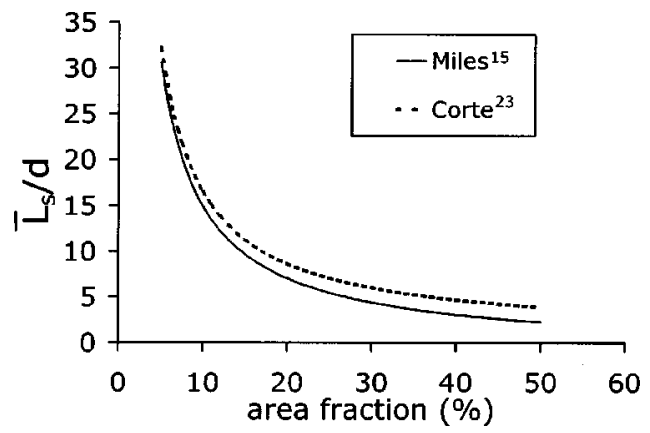

FIG. 5. Mean segment aspect ratio vs area fraction or coverage, $H$ obtained using the formulations of Miles (see Ref. 15) and Corte (see Ref. 23).

$$
-\frac{\pi}{2 \log (1-H)}=\frac{\pi A}{2 N_{f} L_{f} d},
$$

where $N_{f}, L_{f}, d$, and $A$ are properties of the simulated beam network, and $H$ is determined from image analysis of a material. For beams and simulation windows of unit length and side length, Eq. (13) becomes

$$
N_{f} d=-\log (1-H) .
$$

Thus, the diameter of beam elements can be calculated, using the number of beam elements and the area fraction, $H$.

In experimental nanotube sheets, Smith et al. ${ }^{24}$ reported the "empty" volume (i.e., the volume of sheet not occupied by nanotube material) to be $\sim 70 \%-90 \%$. Based on thermogravimetric analysis, Coleman et l. $^{4}$ found the empty volume of a nanotube sheet, prior to polymer intercalation, to be $73 \%$. For a negligible volume fraction of impurities in a sheet, the volume fraction can thus reasonably be approximated to be $\sim 10 \%-30 \%$. Assuming a circular cross section of diameter $d$ for each nanotube rope, the volume fraction of a layer of thickness $d$ of the nanotube sheet can be written as

$$
\text { v.f. }=\frac{N_{f} \pi d L_{f}}{4 A},
$$

where $N_{f}$ and $L_{f}$ are the number and length of a nanotube ropes, and $A$ is the plane area of the sheet. The volume fraction can be rewritten in terms of the area fraction $H$, and $H$ can in turn be expressed as

$$
H=1-e^{-4 / \pi v . f .} .
$$

Using the range of volume fractions given by Smith, ${ }^{24}$ the range of area fractions for unaligned nanotube sheets can be calculated [per Eq. (6)] to be $\sim 12 \%-32 \%$. The measured area fractions for the nanotube sheets studied in this work were between $45 \%$ and $50 \%$; these higher values may have been due in part to 2D/3D imaging artifact (field-of-depth error in SEM).

In our simulations, we used a range of area fractions of $10 \%-50 \%$ in order to bound both reported values in the literature and those obtained by the present authors, from image analysis. For each of value of $H$ considered, the number of beams in each simulation was kept constant and the beam diameter, $d$ was calculated using Eq. (14). 


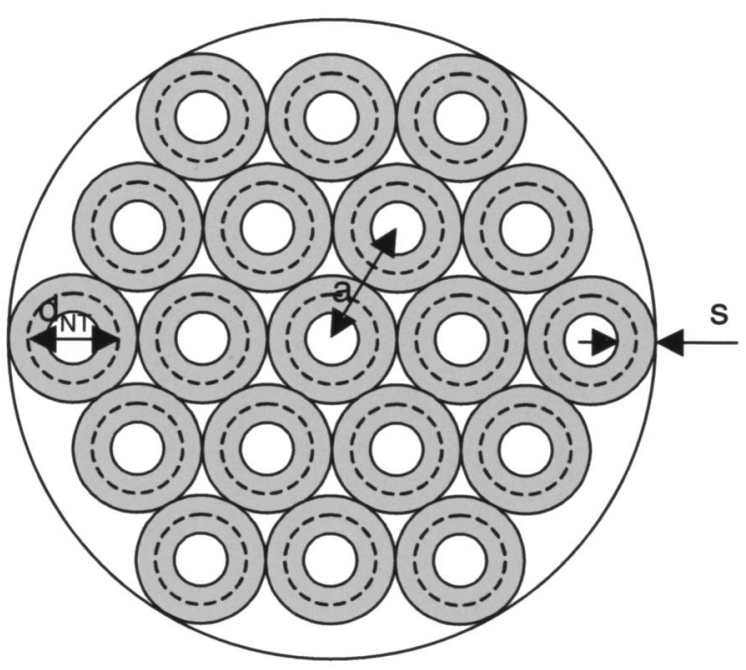

FIG. 6. Hexagonal close packed configuration assumed for nanotubes within a nanorope.

\section{B. Beam geometry}

Nanotube ropes were modeled as porous beams having diameter $d$. Hexagonal close packing of tubes within each rope was assumed as shown in Fig. 6; tubes were modeled as a hollow, circular cross-sectioned cylinders, of wall thickness $0.34 \mathrm{~nm} .{ }^{5,25,26}$ The lattice constant, $a$, was determined from

$$
a=d_{\mathrm{NT}}+s \text {, }
$$

where $d_{\mathrm{NT}}$ is the average nanotube diameter $(0.8 \mathrm{~nm}$ for HiPco-nanotubes; $1.36 \mathrm{~nm}$ for laser ablation-manufactured nanotubes). Per other reported values, an intertube distance of $0.34 \mathrm{~nm}$ was assumed in all cases. ${ }^{25,27}$

Correction factors were used to scale the cross-sectional areas and moments of inertia of each beam. For each real material simulated, the average rope diameter was found using image analysis. The number of tubes $N$, in an average rope was calculated assuming hexagonal close packing. Effective area, $A_{\text {rope }}$ was calculated as the product of $N$ and cross-sectional area of a single tube, $A_{\mathrm{NT}}$. Effective moment of inertia $I_{\text {rope }}$ was found using the parallel axis theorem. Area and moment of inertia correction factors were computed using the ratios $A_{\text {rope }} / A_{\text {solid }}$ and $I_{\text {rope }} / I_{\text {solid }}$, where $A_{\text {solid }}$ and $I_{\text {solid }}$ are the cross-sectional area and moment of inertia of a beam of solid cross section with diameter equal to the rope diameter.

\section{Joint modeling}

Previously observed nanotube joint types are illustrated in Fig. 7, and summarized by type in Table II. Briefly, connections between nanoropes and nanotubes include following distinct types:

- crossed ropes;

- nanosoldered joints, with connecting material possibly comprised of contaminants introduced in the manufacture and purification processes (for example remains from surfactant pyrolysis);

- branched ropes;

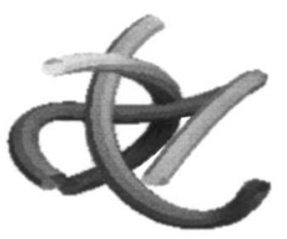

(a)

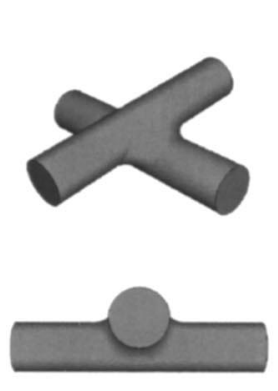

(c)

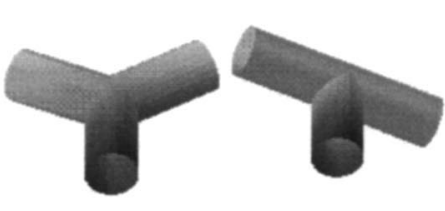

(b)

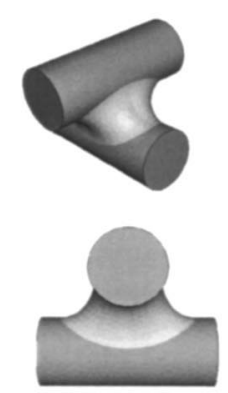

(d)

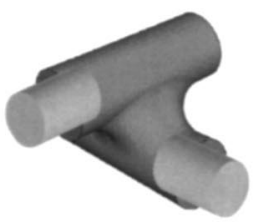

(e)

FIG. 7. Various observed morphologies of joints in nanotube mats, including (a) crossed or entangled ropes (b) $T$ - and $Y$-branched tubes, (c) nanowelded tubes, (d) nanosoldered tubes, and (e) coated tubes/ropes.

- adhered "live" ends of nanotube ropes to the sides of other ropes; and

- coated ropes; with coatings comprised of other materials (e.g., remains of surfactant pyrolysis, or hydrocarbon contaminants).

An expression for degree-of-intersect (d.o.i.) was previously introduced by Berhan and Sastry ${ }^{21}$ to describe the interpenetration of joined fibers. This parameter is defined as the ratio of the beam diameter $d$, to the width of the joint $T$, as shown in Fig. 8. We used this concept in our approach to modeling the various connection scenarios, as summarized in Table III. 3D finite element simulations were performed on two-beam assemblies having joint morphologies defined by d.o.i., ratio of fillet radius to beam radius, and ratio of fillet modulus to beam modulus. A diagram of a typical assembly with joint detail is shown in Fig. 9(a). For each joint, simulations were performed for beam aspect ratios $5<\left(L_{1} / d\right)$ $<15$. For each simulation, the node at one end (" $A$ ") was fixed and a displacement $X$ was applied to the opposite node (" $C$ "). Resultant forces $P_{c}, Q_{c}$, and moment $M_{c}$ were found, and normalized effective modulus was calculated from

$$
\frac{E_{\text {effective }}}{E}=\frac{Q_{c} L_{2}}{\pi r^{2} X E},
$$

where $r$ and $E$ are the beams' radius and Young's modulus, respectively. 
TABLE II. Descriptions of types of nanotube joints, with selected references.

\begin{tabular}{|c|c|c|c|}
\hline Joint & Description & $\begin{array}{l}\text { Selected } \\
\text { references }\end{array}$ & Figure \\
\hline $\begin{array}{l}\text { Crossed/ } \\
\text { entangled } \\
\text { tubes }\end{array}$ & $\begin{array}{l}\text { Load transferred among } \\
\text { crossed and/or entangled } \\
\text { nanotubes via friction and } \\
\text { van der Waals forces }\end{array}$ & $28,29,30$ & $7(\mathrm{a})$ \\
\hline $\begin{array}{l}Y \text { and } T \\
\text { joints }\end{array}$ & $\begin{array}{l}\text { Branched SWNT formed } \\
\text { during nanotube synthesis. } \\
\text { "Live" ends of tubes or } \\
\text { ropes may adhere to the } \\
\text { sides of other tubes/ropes } \\
\text { and resemble } T \text { or } Y \\
\text { joints }\end{array}$ & $31,32,33$ & $7(b)$ \\
\hline Nanowelding & $\begin{array}{l}\text { Irradiation of nanotube } \\
\text { crossing sites leads to } \\
\text { defects in tube structure } \\
\text { which allow tubes to } \\
\text { coalesce }\end{array}$ & 30,34 & $7(\mathrm{c})$ \\
\hline Nanosoldering & $\begin{array}{l}\text { Crossed nanotubes } \\
\text { connected by a deposit of } \\
\text { amorphous carbon with no } \\
\text { coalescence of the tubes }\end{array}$ & 30,35 & $7(\mathrm{~d})$ \\
\hline $\begin{array}{l}\text { Joints } \\
\text { between } \\
\text { coated } \\
\text { tubes or } \\
\text { ropes }\end{array}$ & $\begin{array}{l}\text { Indivdual tubes and/or } \\
\text { ropes coated with by- } \\
\text { products of tube synthesis } \\
\text { or processing may be } \\
\text { joined by a bond between } \\
\text { the coatings of the } \\
\text { individual tubes/ropes }\end{array}$ & 35 & $7(\mathrm{e})$ \\
\hline
\end{tabular}

The horizontal resultant force at $C, Q_{c}^{\prime}$, was calculated using beam theory, following the same method as previously for the compliant zone model..$^{20,21}$ This force was calculated for each $2 \mathrm{D}$ torsion spring joined beam having the same values of $L_{1}, L_{2}, d, E$, and $X$, as for each 3D simulation [Fig. 9(b)]. By setting $Q_{c}=Q_{c}^{\prime}$, and solving for $K$, the value of the torsion spring constant required to produce the same

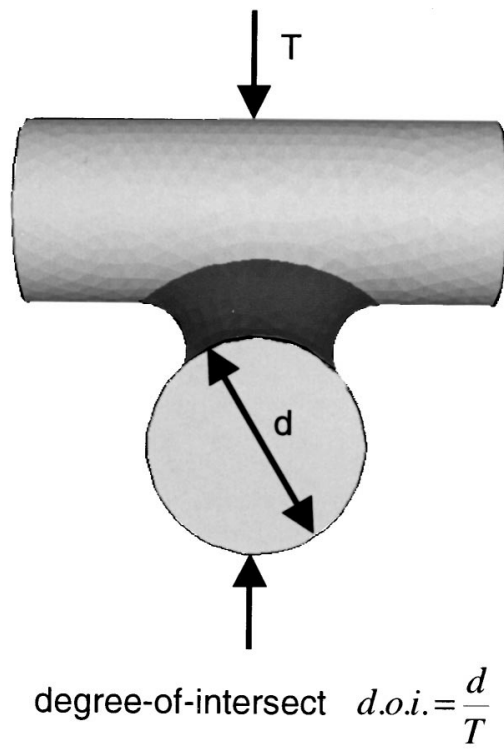

FIG. 8. Definition of d.o.i.
TABLE III. Approach to 3D modeling of various nanotube joint configurations.

\begin{tabular}{lccl}
\hline \hline Joint & d.o.i. & $\begin{array}{c}\text { 2D } \\
\text { model }\end{array}$ & \multicolumn{1}{c}{ 3D model } \\
\hline $\begin{array}{l}\text { Crossing/ } \\
\text { entangled } \\
\text { ropes }\end{array}$ & 0.5 & $\begin{array}{c}\text { Torsion } \\
\text { spring }\end{array}$ & Contact elements \\
$\begin{array}{l}Y \text { and } T \\
\text { joints }\end{array}$ & 1 & $\begin{array}{l}\text { Rigid } \\
\text { bond }\end{array}$ & $\begin{array}{l}\text { Filleted joint with fillet } \\
\text { material same as that of } \\
\text { the connected beams }\end{array}$ \\
Nanowelding & $0.5-$ & $\begin{array}{l}\text { Rigid } \\
\text { bond }\end{array}$ & $\begin{array}{l}\text { Filleted joint with fillet } \\
\text { material same as that of } \\
\text { the connected beams }\end{array}$ \\
$\begin{array}{l}\text { Nanosoldering } \\
\text { Joining of } \\
\text { coated } \\
\text { tubes or } \\
\text { ropes }\end{array}$ & $\leqslant 0.5$ & $\begin{array}{l}\text { Torsion } \\
\text { spring }\end{array}$ & $\begin{array}{l}\text { Filleted joint with fillet } \\
\text { material different from that } \\
\text { of the connected beams } \\
\text { Filleted joint with fillet and } \\
\text { coating modeled as a } \\
\text { material different from that } \\
\text { of the connected beams }\end{array}$ \\
\hline \hline
\end{tabular}

effective modulus for the $2 \mathrm{D}$ and $3 \mathrm{D}$ cases was found. This torsion spring constant was normalized to give the rigidity parameter, $\bar{K}$, using the normalization ${ }^{36}$

$$
\bar{K}=\frac{K L_{1}}{E I+K L_{1}} .
$$

This normalization replaced our earlier convention, $\bar{K}$ $=K L / E I,{ }^{17,19,21}$ since it scales the rigidity of the joint to be between zero and one, representing pin-jointed and rigid connections, respectively.

Figure 10 shows a plot of the rigidity parameters obtained for the seven joint morphologies considered, versus

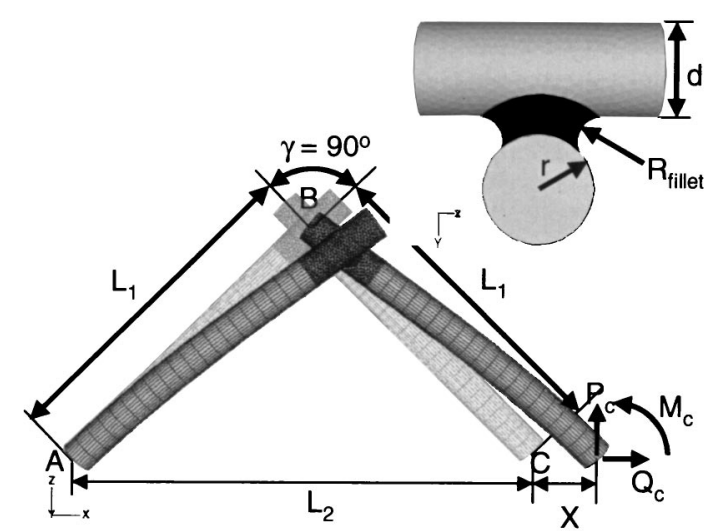

(a)

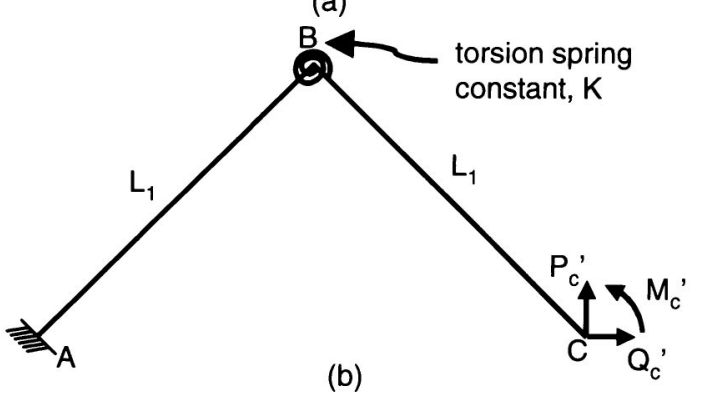

(b)

FIG. 9. Modeling of joints between nanotubes, including (a) 3D finite element joint model, used to inform a (b) 2D torsion spring-jointed model. 


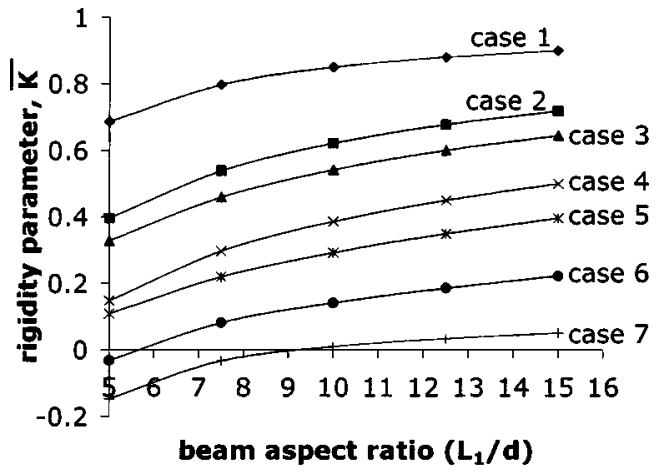

FIG. 10. Plot of rigidity parameter vs beam aspect ratio for the seven different joint morphologies examined (Table IV).

beam aspect ratio. Table IV describes the joints defined as cases 1-7. Figure 10 confirms that a range of $3 \mathrm{D}$ joint morphologies can readily be represented by suitable torsion spring constants in 2D beam models. However, for some combinations of beam aspect ratio and joint architecture, an equivalent linear torsion spring cannot be found, and a restoring moment is required at the joint of the $2 \mathrm{D}$ model in order to produce the same response as the 3D joint, as evidenced by the negative rigidity parameters obtained for some of the cases considered.

\section{Finite element simulations: Networks}

In the network finite element analyses, joints were modeled as torsion springs to simulate the various joint morphologies. All torsion springs in a given simulation were assigned the same rigidity parameter, $\bar{K}$, with $L_{1}$ in Eq. (19) replaced by $\bar{L}_{s}$. For each network, a MATLAB finite element analysis was performed for rigidity parameters 0.05 , 0.1 , and 1 (with 1 representing the rigid bond case). EulerBernoulli beam elements having torsion springs at each end were used, following Kassimali's ${ }^{36}$ formulation for beams with semirigid joints. The local torsion spring constants for the springs at the end of each element were chosen such as to achieve the desired rigidity parameter at each joint of the network.

Random beam networks were generated as shown in Fig. 3 . The nodes on the bottom boundary $(y=0)$ were fixed, and those on the top boundary $\left(y=L_{u}\right)$ were given a displacement $d y=0.001$ in the $y$ direction. The total resultant force in the $y$ direction on the top boundary, $F_{y^{\prime}}$ was found for each simulation. The effective modulus, $E_{\text {effective }}$ was then calculated from

TABLE IV. Description of 3D joints modeled in finite element simulations.

\begin{tabular}{clcc}
\hline \hline Case No. & d.o.i. & $R_{\text {fillet }} / r$ & $E_{\text {fillet }} / E$ \\
\hline 1 & 0.465 & 1.0 & 0.10 \\
2 & 0.465 & 0.5 & 0.10 \\
3 & 0.5 & 0.1 & 1.00 \\
4 & 0.465 & 1.0 & 0.01 \\
5 & 0.465 & 0.3 & 0.10 \\
6 & 0.465 & 0.5 & 0.01 \\
7 & 0.465 & 0.3 & 0.01 \\
\hline \hline
\end{tabular}

$$
E_{\text {effective }}=\frac{F_{y} L_{u}}{d L_{u} d y},
$$

where $d$ is the beam diameter, and $L_{u}$ is the length of the sides of the simulation window ( $L_{u}=1$ for the unit cell). The values of $E_{\text {effective }}$ were then normalized by the beam Young's modulus, $E$. Finite element analyses were performed on networks of 200 fibers, with area fractions $H$ of $10 \%, 20 \%$, $30 \%, 40 \%$, and $50 \%$, respectively. Ten networks were generated and analyzed for each area fraction.

\section{EXPERIMENTAL APPROACH}

\section{A. Materials manufacture}

Free-standing SWNT sheets were made by filtration of SWNT dispersions using Triton X-100 surfactant (Aldrich). ${ }^{10}$ Purified SWNTs produced by both the pulsed laser vaporization technique ${ }^{37}$ (Tubes@Rice, lot No. R062900), and purified as described by Rinzler et al. ${ }^{1}$ and the HiPco (highpressure $\mathrm{CO}$ disproportionation) ${ }^{38}$ method (Carbon Nanotechnologies, Inc., lot No. CM26-0024-1, in the form of BuckyPearls ${ }^{\mathrm{TM}}$ ) were employed in this work. By applying vacuum or, alternatively, pressure (500 $\mathrm{kPa}$ of nitrogen), the SWNT dispersions were passed through Millipore Mitex 5 $\mu \mathrm{m}$ LS membrane filters. The resulting sheets were extensively washed with water and methanol to remove the surfactant, and dried. Thermal annealings comprising 9 hour long temperature ramps to $1000^{\circ} \mathrm{C}$, followed by $10 \mathrm{~min}$ residence times, were carried out to remove residual surfactant and solvent.

\section{B. Mechanical testing}

The mechanical properties of the carbon nanotube sheets were measured using a dynamic mechanical analyzer Perkin Elmer DMA7e. $1 \mathrm{~mm}$ wide strips were tested at room temperature, byapplying an initial force of $5 \mathrm{mN}(15 \mathrm{mN}$ for sample No. 84 annealed) and a static stress rate of $20 \mathrm{mN} /$ $\min$. The papers were tested using a static stress test with the force increasing at a rate of $20 \mathrm{mN}$ per min. The probe height and the applied force were determined every $0.6 \mathrm{~s}$, from which stress and strain were calculated. Samples were directly deposited onto conducting carbon tape, and imaged using a LEO 1530 VP scanning electron microscope.

\section{RESULTS}

\section{A. Simulation results}

Figure 11 illustrates the effect of altering the rigidity parameter on effective modulus, in simulations of 200 fiber HiPco sheets. Points represent mean values of $E_{\text {effective }} / E$ for ten simulations at each area fraction, and the errors bars shown are for $\pm 1 \sigma$.

\section{B. Comparison with experimental results}

Stress-strain plots obtained for the five samples tested are shown in Fig. 12; numerical values of Young's moduli, maximum strains, tensile strengths, and the area fractions are presented in Table V. Experimentally determined Young's moduli were normalized with that of the constituent ropes. 


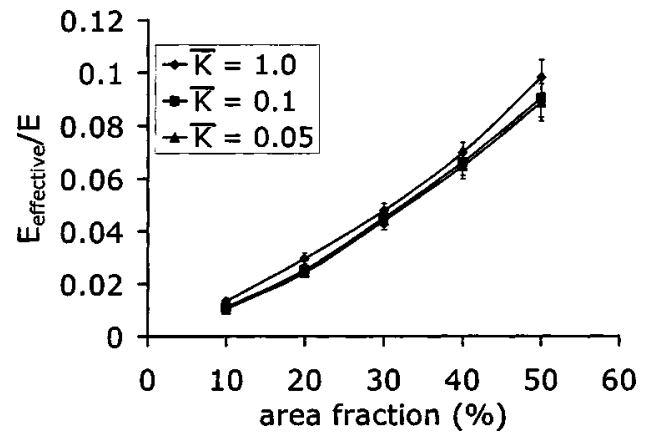

FIG. 11. Plots of normalized effective modulus vs area fraction for HiPco sheets, for $\bar{K}=1,0.1$ and 0.05 . An average rope diameter $10 \mathrm{~nm}$ was assumed in all cases.

The latter value was adopted from $\mathrm{Lu},{ }^{5}$ who used an empirical force constant model to obtain the elastic properties of nanoropes comprised of single walled carbon nanotubes of various radii. For the constituent ropes of the HiPco and laser ablation sheets we used $E=0.736$ and $0.563 \mathrm{TPa}$, which are the values of Young's modulus for ropes comprised of tubes of radius 0.4 and $0.67 \mathrm{~nm}$, respectively, as reported by $\mathrm{Lu}^{5}$ Final comparisons of the normalized experimental results with the Euler beam/rigid bond simulations for 200-fiber HiPco and laser ablation sheets are shown in Fig. 13.

\section{DISCUSSION}

Figure 13 shows the effective modulus of the nanotube obtained from 2D Euler beam simulations using both Corte' ${ }^{23}$ and Miles ${ }^{15}$ expressions for area fraction and number of fiber crossings per unit area. The difference between the two approaches becomes more significant as the area fraction is increased. Miles' ${ }^{15}$ formulation is more accurate since he takes into account the overlapping area of fibers at crossing points. We thus take these as the upper bounds on achievable modulus for the nanotube sheets.

A plot of $\bar{L}_{s} / d$ vs $H$ (Fig. 5) shows that for $H>27 \%$ the mean segment aspect ratio is less than 5 . For this range of area fractions, therefore, simulations using Timoshenko, rather than Euler beam elements would be more appropriate. However, implementation of Timoshenko beam theory

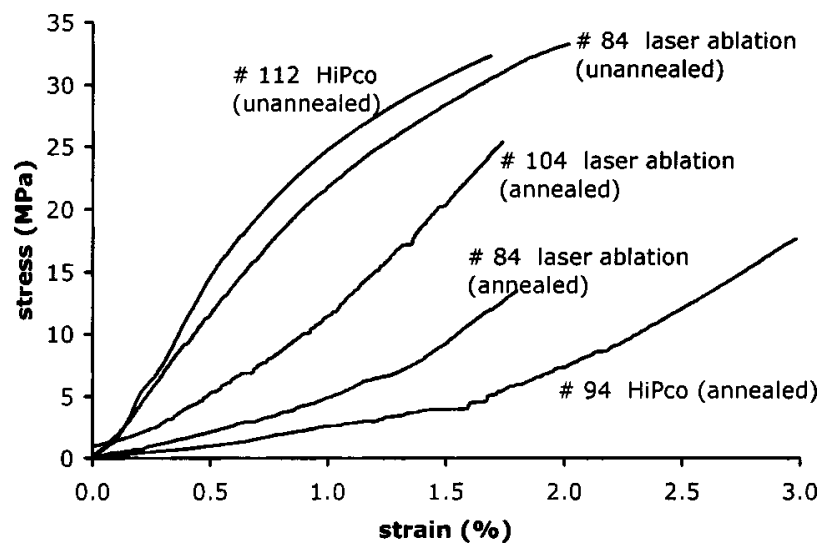

FIG. 12. Stress vs strain results of mechanical tests on nanotube sheet samples.
TABLE V. Description and mechanical properties of nanotube sheet samples used.

\begin{tabular}{|c|c|c|c|c|}
\hline \multirow[b]{2}{*}{ Sample No. } & \multirow[b]{2}{*}{ Description } & \multicolumn{2}{|c|}{ Average diameter (nm) } & \multirow{2}{*}{$\begin{array}{l}\text { Area } \\
\text { fraction } \\
(\%)\end{array}$} \\
\hline & & Nanorope & Nanotube & \\
\hline 84 & $\begin{array}{l}\text { Laser ablation } \\
\quad \text { (annealed) }\end{array}$ & $\sim 10$ & 1.36 & 45 \\
\hline 84 & $\begin{array}{c}\text { Laser ablation } \\
\text { (unannealed) }\end{array}$ & $\sim 10$ & 1.36 & 49 \\
\hline 94 & $\begin{array}{c}\text { HiPco } \\
\text { (annealed) }\end{array}$ & $\sim 10$ & 0.8 & 48 \\
\hline 112 & $\begin{array}{c}\text { HiPco } \\
\text { (unannealed) }\end{array}$ & $\sim 10$ & 0.8 & 46 \\
\hline 104 & $\begin{array}{l}\text { Laser ablation } \\
\quad \text { (annealed) }\end{array}$ & $\sim 10$ & 1.36 & 45 \\
\hline
\end{tabular}

would require knowledge of the shear modulus of the nanotube ropes, as well as a model for the load transfer mechanism among the tubes of the rope so that an appropriate shear correction factor could be selected. At this time, the load transfer mechanism within nanotube ropes is still the subject of intensive investigation; shear moduli values reported in the literature differ by as much as an order of magnitude. ${ }^{39,40}$ Even if there were broad agreement on the values, however, the effective moduli obtained from use of Timoshenko beam elements would be lower than those obtained from Euler beam elements.

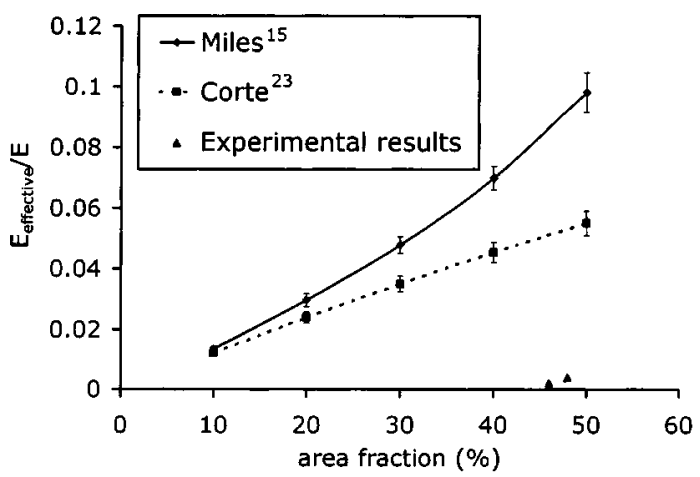

(a)

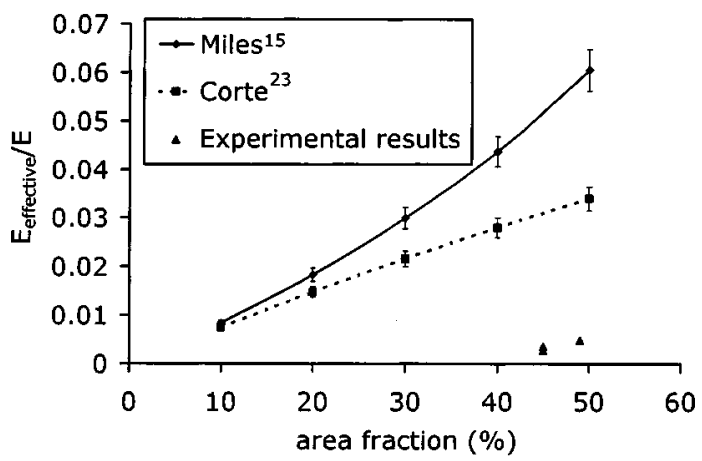

(b)

FIG. 13. Comparison of experimental and simulation results for normalized effective modulus, for nanotube sheets containing nanotubes synthesized by the (a) HiPco and (b) laser ablation processes. Simulation results derived using both Miles (see Ref. 15) and Corte (see Ref. 23) are shown. An average rope diameter $10 \mathrm{~nm}$ was assumed in all cases. 
Also in keeping with our aim to determine upperbound properties, we selected correction factors to scale crosssectional areas and moments of inertia in beams, by assuming equal load sharing among the tubes within a rope. We thus modeled conditions wherein nanotubes within ropes were physically bonded, as might occur with irradiation or chemical treatment of the ropes. Recent experiments on SWNTs $^{40}$ and composites comprised of SWNTs $^{41}$ have pointed to intertube sliding as a mechanism for load sharing in a bundle, which would certainly lower resulting moduli.

Our experimental moduli fell well below upper bound predictions (as shown in Fig. 13), as expected. Further, comparison of Fig. 13(a) and Fig. 11 show the experimental results fell well below the results for simulations with a low rigidity parameter of 0.05 , suggesting that the nanoropes are physically connected at some crossing points, and not at all at others.

We reiterate the distinction between fiber crossing and fiber bond: though a statistically based prediction of fiber crossings is available for $2 \mathrm{D}$ networks, it may not comprise the actual density of fiber bonds, if crossing fibers do not always fuse. Specifically, if nanoropes are connected at a fraction, $b$, of crossings, then the mean segment length is increased by a factor of $1 / b$, and the corresponding value of maximum normalized modulus is shifted to the value given at area fraction $b \cdot H$. The upper bounds for effective moduli obtained here (Fig. 13) suggest that optimization of processing of nanotube sheets, to produce connections at each and every fiber crossing, could increase effective moduli by a factor of $\sim 2.5-30$. For example, if the area fractions estimated from image analysis are correct, the effective moduli of the laser and HiPco sheets can be increased by $\sim 15$ times and $\sim 32$-fold, respectively, if a connection were made at every crossing.

Finally, if the actual volume fractions of the sheets tested were within the range given by $\mathrm{Smith}^{24}$ of between $10 \%$ and $30 \%$ (i.e., if area fractions obtained from image analysis were an overestimation), we must revise the potential improvement in nanotube mat properties. If this is the case, the effective moduli of the laser sheets can potentially be increased by a factor between 2.5 and 8 , and that of the HiPco sheets by between $5 \%$ and $16 \%$.

\section{CONCLUSIONS/FUTURE WORK}

In this work we present a method for calculating upper bounds on the Young's moduli of nanotube sheets comprised of randomly oriented nanotube ropes. Similar methods could be employed to obtain upper bounds on the moduli of films comprised of aligned ropes. Experimental moduli fall significantly below simulated upper bounds, indicating that there is still room for improvement in manufacturing, to realize higher bond densities and thus higher moduli. However, even the maximum achievable moduli are still rather low as compared to the Young's moduli of the nanotubes themselves. The upper bounds are therefore useful in determining whether the modulus desired for a particular application is attainable.
While the effects of joint morphology and beam model have been interrogated in this work, the rope segments were modeled as straight beams. The inherent waviness of the nanotube ropes would certainly reduce the sheet modulus, and may be a separate feature which can be optimized in sheet manufacture. This factor will be addressed in future work.

\section{ACKNOWLEDGMENTS}

The authors gratefully acknowledge support for this work from the Defense Advanced Research Projects Agency (DARPA) the W.M. Keck Foundation, Synthetic Multifunctional Materials Program (Dr. Leo Christodoulou, Program Manager, DARPA-DSO, and Dr. Steve Fishman, Program Manager, Office of Naval Research) and the National Science Foundation (NSF), through a PECASE (Sastry).

${ }^{1}$ A. G. Rinzler et al., Appl. Phys. A: Mater. Sci. Process. 67, 29 (1998).

${ }^{2}$ R. H. Baughman et al., Science 284, 1340 (1999).

${ }^{3}$ A. Minett, J. Fraysse, G. Gang, G. T. Kim, and S. Roth, Current Applied Physics 2, 61 (2002).

${ }^{4}$ J. N. Coleman et al., Appl. Phys. Lett. 82, 1682 (2003).

5 J. P. Lu, Phys. Rev. Lett. 79, 1297 (1997).

${ }^{6}$ T. V. Sreekumar, T. Liu, S. Kumar, L. M. Ericson, R. H. Hauge, and R. E. Smalley, Chem. Mater. 15, 175 (2003).

${ }^{7}$ B. Vigolo, A. Penicaud, C. Coulon, C. Sauder, R. Pailler, C. Journet, P. Bernier, and P. Poulin, Science 290, 1331 (2000).

${ }^{8}$ P. Poulin, B. Vigolo, and P. Launois, Carbon 40, 1741 (2002).

${ }^{9}$ Y. H. Li, J. Q. Wei, X. F. Zhang, C. L. Xu, D. H. Wu, L. Lu, and B. Q. Wei, Chem. Phys. Lett. 365, 95 (2002)

${ }^{10}$ J. Liu et al., Science 280, 1253 (1998).

${ }^{11}$ H. L. Cox, Br. J. Appl. Phys. 3, 72 (1952).

${ }^{12}$ W. T. Lu and L. A. Carlsson, Tappi J. 79, 203 (1996).

${ }^{13}$ O. Kallmes and H. Corte, Tappi J. 43, 737 (1960).

${ }^{14}$ S. Goudsmit, Rev. Mod. Phys. 17, 321 (1945).

${ }^{15}$ R. E. Miles, Proc. Natl. Acad. Sci. U.S.A. 52, 901 (1964).

${ }^{16}$ R. E. Miles, Proc. Natl. Acad. Sci. U.S.A. 52, 1157 (1964).

${ }^{17}$ A. M. Sastry, C. W. Wang, and L. Berhan, Probabilistic Methods in Fatigue and Fracture 200, 229 (2001).

${ }^{18}$ X. Cheng, C. Wang, A. M. Sastry, and S. B. Choi, J. Eng. Mater. Technol. 121, 514 (1999).

${ }^{19}$ C. W. Wang and A. M. Sastry, J. Eng. Mater. Technol. 122, 460 (2000).

${ }^{20}$ C. W. Wang, L. Berhan, and A. M. Sastry, J. Eng. Mater. Technol. 122, 450 (2000).

${ }^{21}$ L. Berhan and A. M. Sastry, J. Compos. Mater. 37, 715 (2003).

${ }^{22}$ Y.-B. Yi, L. Berhan, and A. M. Sastry (unpublished).

${ }^{23} \mathrm{H}$. Corte, in Structure, Solid Mechanics and Engineering Design. The Proceedings of the Southampton 1969 Civil Engineering Materials Conference, Part I, edited by M. Te'eni (Wiley, New York, 1969), pp. 341355.

${ }^{24}$ B. W. Smith, Z. Benes, D. E. Luzzi, J. E. Fischer, D. A. Walters, M. J. Casavant, J. Schmidt, and R. E. Smalley, Appl. Phys. Lett. 77, 663 (2000).

${ }^{25}$ M. F. Yu, B. S. Files, S. Arepalli, and R. S. Ruoff, Phys. Rev. Lett. 84, $5552(2000)$

${ }^{26}$ V. M. Harik, Comput. Mater. Sci. 24, 328 (2002).

${ }^{27}$ J. Tersoff and R. S. Ruoff, Phys. Rev. Lett. 73, 676 (1994).

${ }^{28}$ M. S. Fuhrer et al., Science 288, 494 (2000).

${ }^{29}$ Y. G. Yoon, M. S. C. Mazzoni, H. J. Choi, J. Ihm, and S. G. Louie, Phys. Rev. Lett. 86, 688 (2001).

${ }^{30}$ H. Nishijima, S. Akita, and Y. Nakayama, Jpn. J. Appl. Phys., Part 1 38, 7247 (1999)

${ }^{31}$ M. Menon and D. Srivastava, J. Mater. Res. 13, 2357 (1998).

${ }^{32}$ D. Zhou and S. Seraphin, Chem. Phys. Lett. 238, 286 (1995).

${ }^{33}$ P. Lambin, A. Fonseca, J. P. Vigneron, J. B. Nagy, and A. A. Lucas, Chem. 
Phys. Lett. 245, 85 (1995).

${ }^{34}$ M. Terrones, F. Banhart, N. Grobert, J. C. Charlier, H. Terrones, and P. M. Ajayan, Phys. Rev. Lett. 89, 075505 (2002).

${ }^{35}$ F. Banhart, Nano Lett. 1, 329 (2001).

${ }^{36}$ A. Kassimali, Matrix Analysis of Structures (Brooks/Cole, Pacific Grove, CA, 1999).

${ }^{37}$ A. Thess et al., Science 273, 483 (1996).
${ }^{38}$ P. Nikolaev, M. J. Bronikowski, R. K. Bradley, F. Rohmund, D. T. Colbert, K. A. Smith, and R. E. Smalley, Chem. Phys. Lett. 313, 91 (1999).

${ }^{39}$ R. B. Pipes and P. Hubert, Nano Lett. 3, 239 (2003).

${ }^{40}$ J. P. Salvetat, G. A. D. Briggs, J. M. Bonard, R. R. Bacsa, A. J. Kulik, T. Stockli, N. A. Burnham, and L. Forro, Phys. Rev. Lett. 82, 944 (1999).

${ }^{41}$ P. M. Ajayan, L. S. Schadler, C. Giannaris, and A. Rubio, Adv. Mater. (Weinheim, Ger.) 12, 750 (2000). 